\title{
Prognostic Implication of Preoperative Serum Albumin to Carcinoembryonic Antigen Ratio in Colorectal Cancer Patients
}

\section{Mingyue Xu}

Hainan Hospital of Chinese PLA General Hospital

\section{You Liu}

Hainan Hospital of Chinese PLA General Hospital

Tianhui Xue

Hainan Hospital of Chinese PLA General Hospital

Qianwen Ye

Hainan Hospital of Chinese PLA General Hospital

Jia Xiang

Hainan Hospital of Chinese PLA General Hospital

\section{Long Liu}

Tongji Hospital Affiliated to Tongji University: Shanghai Tongji Hospital

Bing Yan ( $\nabla$ y_bing41@163.com )

Hainan Branch of PLA General Hospital https://orcid.org/0000-0001-5430-4761

\section{Research Article}

Keywords: Colorectal cancer, Albumin, Carcinoembryonic antigen, Progression-free survival, Overall survival

Posted Date: September 10th, 2021

DOI: https://doi.org/10.21203/rs.3.rs-874216/v1

License: (c) (1) This work is licensed under a Creative Commons Attribution 4.0 International License. Read Full License 


\section{Abstract}

Both preoperative serum albumin (ALB) and carcinoembryonic antigen (CEA) were previously reported as useful prognostic factors in colorectal cancer (CRC); however, the ALB to CEA ratio (ACR) and their individual prognostic efficacies have been less studied. In this study, a total of 156 CRC patients staged IIV were retrospectively enrolled. Patients were divided into ACR-low or ACR-high subgroups, and the differences in progression-free survival (PFS) and overall survival (OS) were conducted by Kaplan-Meier curves, log-rank test and Cox proportional model. As a result, a total of 31.41\% (49/156) of patients presented with ACR-low disease, and these patients had tumors with advanced T stages $\left(T_{3}+T_{4}\right)$ $(P<0.01)$, larger tumor diameters $(P<0.01)$ and distant metastases $(P=0.01)$ and a relatively lower lymphocyte to monocyte ratio $(L M R)(P<0.01)$. The ACR was significant in predicting survival. When 5.98 was used as the cutoff point, it had a sensitivity of $58.50 \%$ and $61.50 \%$ and a specificity 0 f $83.50 \%$ and $80.50 \%$ for PFS and OS, respectively. ACR displayed a superior prognostic efficacy than other tested markers for both PFS and OS (except LMR). Patients in the ACR-low group displayed significantly worse PFS (log rank $=35.75, \mathrm{P}<0.01)$ and OS $(\log$ rank $=29.68, \mathrm{P}<0.01)$ than those in the ACR-high group . Finally, ACR was an independent prognostic factor for both PFS (HR $=0.31,95 \% \mathrm{Cl}: 0.17-0.56, \mathrm{P}<0.01)$ and $\mathrm{OS}(\mathrm{HR}=0.33,95 \% \mathrm{Cl}: 0.16-0.66, \mathrm{P}<0.01)$. For conclusion, the ACR was a robust prognostic factor in $\mathrm{CRC}$, and patients with a relatively low preoperative ACR had significantly worse survival.

\section{Introduction}

Colorectal cancer (CRC) is a classic solid tumor that causes many deaths worldwide[1], including in China[2]. Although early diagnosed cases could be cured by surgery, the majority of advanced cases are still incurable [3]. Identifying simple and reliable prognostic factors in the disease has become increasingly popular in recent years.

Albumin (ALB) acts as a carrier for practically all metabolites, drugs, hormones and products of toxic degradation in the human body[4], and its metabolism is affected by many exogenous and endogenous factors, including cancer cells[5]. Previously, several studies demonstrated the usefulness of individual ALB as a prognostic factor in many malignancies, including gastric cancer [6], nonsmall cell lung cancer [7], ovarian cancer [8], head and neck cancer [9] and, in particular, CRC [10-13]. Notably, the prognostic efficacy of ALB alone was inferior when compared with other markers[11]. In recent years, some innovative markers based on ALB, such as the albumin to globulin ratio[14-15], albumin to fibrinogen ratio[16], C-reactive protein to albumin ratio (CAR)[17-19] and others[20-22], have been frequently reported in cancer studies, including CRC. However, some of these parameters are not conventional prognostic factors and are not included in routine clinical tests, and more common markers are still needed.

Carcinoembryonic antigen (CEA) is a member of a family of glycoproteins anchored to the cell membrane of colonic epithelial cells and up to approximately $90 \%$ of colorectal cancer cells can release CEA $[23,24]$. CEA is also a long-term established marker in the diagnosis, surveillance and prognosis of CRC. 
Regrettably, only approximately $1 / 3$ of patients have an abnormally elevated CEA at the time of diagnosis[25], which largely impairs its application in clinical practice. Interestingly, some investigators have attempted to further explore the prognostic value of CEA in patients whose levels fall into the normal range before surgery[26, 27]; however, the cutoff points were not consistent, and its value in postoperative surveillance in such a scenario was still mainly unclear. These studies also indicated that a single CEA assay was still insufficient for predicting patients' prognosis. Taking into consideration these results, it was plausible that combining ALB and CEA would have potential prognostic value in CRC, but studies addressing this topic have not been published.

In this study, we aimed to explore the prognostic value of the ALB to CEA ratio (ACR) as well as its prognostic efficacy when compared with individual ALB, CEA and other systemic inflammation markers in CRC.

\section{Materials And Methods}

\section{Patient enrollment}

From January 2011 to January 2018, patients with CRC treated in Hainan Hospital of Chinese PLA General Hospital were enrolled. Those meeting the following criteria were included: 1 . age $>18 \mathrm{y} ; 2$. radical recession of the primary lesion; and patients were excluded based on any one of the following criteria: 1 . neoadjuvant therapies; 2 . missing laboratory tests within a week before surgery or key information in postoperative pathological reports; 3 . multiple or recurrent malignancies or in situ lesions; 4 . abnormal aminotransferase or bilirubin by liver function tests; and 5 . loss to follow-up of less than $36 \mathrm{~m}$. Other clinicopathological parameters were collected as previously reported [28,29], and the body mass index was calculated as body weight $(\mathrm{kg})$ divided by height $\left(\mathrm{m}^{2}\right)$. Tumor size was cut off at $4 \mathrm{~cm}$ according to a previous report [30]. The study was approved by the ethics committee of Hainan Hospital of PLA General Hospital (ID: 301HLFYLS15), and written informed consent was received from the patients or their authorized relatives.

\section{Determination of ACR and other systemic inflammation markers}

Laboratory tests were performed between 6:00 and 9:00 am on peripheral venous blood within 1 week to 1 month before the surgery, and the latest results were used when more than one result was found. The ACR was calculated as ALB (Ref: $35-50 \mathrm{~g} / \mathrm{L}$ ) divided by CEA (Ref: $0-55 \mathrm{ng} / \mathrm{mL}$ ) and then by $10^{6}$ to make the results easier to read. In addition, other systemic inflammation markers, including the neutrophil to lymphocyte ratio (NLR)[31], lymphocyte to monocyte ratio (LMR)[32] and platelet to lymphocyte ratio (PLR)[33], were also determined according to previous reports.

\section{Definition of progression-free survival (PFS) and overall survival (OS)}

The follow-up was conducted by telephone, WeChat or by visit to the medical records at the hospital with an interval of 3-6 months for the first 2 years and 6-12 months for the following years. PFS was defined 
as the point from the date of surgery until the date of first recurrence at any location, disease progression by imaging examinations according to the RECIST (version 1.1) [34] or death from any cause. OS was defined as the date of surgery until the date of death by any cause. The latest follow-up point was June 2021.

\section{Statistical analysis}

The statistical analyses were performed using SPSS 20.0 (SPSS Inc., Chicago, IL, USA). A receiver operating characteristic (ROC) curve analysis was used to determine the optimal discriminator value of ACR, and comparison of the areas under the curve (AUCs) was achieved by MedCalc v19.0.7 (MedCalc Software Ltd., Ostend, Belgium). The differences in clinicopathological parameters in the ACR subgroups were calculated by $\chi^{2}$-test or Fisher's exact test and Student's $t$ when appropriate. Survival differences for ACR-low or high groups were conducted by Kaplan-Meier (K-M) analysis followed by log-rank tests. Risk factors for survival were estimated by a Cox proportional hazards model. Double-sided $\mathrm{P}<0.05$ was considered statistically significant.

\section{Results}

\section{Demographic characteristics and the prognostic efficacy of ACR}

As shown in Fig. 1, a total of 294 patients were registered, and 156 patients (100 males, 56 females) were ultimately included in the study. The mean age of the patients was $60.36 \mathrm{y}$ (range: $24-85 \mathrm{y}$ ), and the mean follow-up was $47.61 \mathrm{~m}$ (range: 1-102 m). According to the ROC tests, ACR was significant for predicting PFS (AUC $=0.75,95 \% \mathrm{Cl}: 0.368-0.83, \mathrm{P}<0.01)$ and OS (AUC $=0.74,95 \% \mathrm{Cl}: 0.65-0.83, \mathrm{P}<$ 0.01 ) (Fig. 2), and when 5.98 (according to the Youden index) was used as the cutoff point, it had a sensitivity of $58.50 \%$ and $61.50 \%$ and a specificity of $83.50 \%$ and $80.50 \%$ for PFS and OS, respectively. Next, further comparison of the prognostic efficacy of ACR with other markers indicated that ACR had a superior efficacy than individual ALB $(Z=2.58, P=0.01), C E A(Z=2.17, P=0.03)$ and $N L R(Z=2.63, P=$ $0.01), \operatorname{LMR}(Z=2.08, P=0.01), P L R(Z=3.03, P<0.01)$ in $P F S$; and it also had a similar advantage over single ALB $(Z=2.05, P=0.04), C E A(Z=2.04, P=0.04)$ and NLR $(Z=2.29, P=0.02), P L R(Z=2.40, P=$ $0.02)$ in OS except $L M R(Z=1.72, P=0.09)$.

\section{Differences in clinicopathological parameters in the ACR-low or ACR-high subgroups}

By ROC tests, the patients were subsequently divided into ACR-low $(<5.98)$ or ACR-high $(\geq 5.98)$ subgroups, and it was found that $31.41 \%(49 / 156)$ of cases were ACR-low.

As shown in Table 1, these patients apparently presented with advanced $T$ stages $\left(T_{3}+T_{4}\right)(P<0.01)$, larger tumor diameters $(P<0.01)$ and distant metastases $(P=0.01)$ and a relatively lower $L M R(P<0.01)$. 
Table 1

Different ACR among varied clinicopathological parameters

\begin{tabular}{|c|c|c|c|c|}
\hline & & ACR & & \\
\hline & Patient No. & $<5.98$ & $\geq 5.98$ & $P$ \\
\hline Age $(y)$ & & & & 0.08 \\
\hline$<60$ & 68 & 16 & 52 & \\
\hline$\geq 60$ & 88 & 33 & 55 & \\
\hline Gender & & & & 0.47 \\
\hline Female & 56 & 20 & 36 & \\
\hline Male & 100 & 29 & 71 & \\
\hline Tumor location & & & & 1.00 \\
\hline Right & 36 & 11 & 25 & \\
\hline Left & 110 & 38 & 82 & \\
\hline Histological grade & & & & 0.12 \\
\hline Well + moderate & 127 & 36 & 91 & \\
\hline Poor & 29 & 13 & 16 & \\
\hline Invasive depth & & & & $<0.01^{*}$ \\
\hline $\mathrm{T}_{1+2}$ & 34 & 4 & 30 & \\
\hline $\mathrm{T}_{3+4}$ & 122 & 45 & 77 & \\
\hline Tumor diameter $(\mathrm{cm})$ & & & & $<0.01^{*}$ \\
\hline$<4$ & 64 & 12 & 52 & \\
\hline$\geq 4$ & 92 & 37 & 55 & \\
\hline Node involvement & & & & 0.23 \\
\hline $\mathrm{N}_{0}$ & 91 & 25 & 66 & \\
\hline$N_{1+2}$ & 65 & 24 & 41 & \\
\hline Positive nodes no. & 156 & $3.12 \pm 5.78$ & $1.64 \pm 3.88$ & 0.15 \\
\hline Distant metastasis & & & & $0.01^{*}$ \\
\hline
\end{tabular}




\begin{tabular}{|c|c|c|c|c|}
\hline \multicolumn{5}{|c|}{ ACR } \\
\hline Yes & 12 & 8 & 4 & \\
\hline No & 144 & 41 & 103 & \\
\hline TNM stage & & & & 0.17 \\
\hline $\mid+\|$ & 87 & 23 & 64 & \\
\hline III + IV & 69 & 26 & 43 & \\
\hline Adjuvant ther & & & & 0.02 \\
\hline Received & 85 & 31 & 54 & \\
\hline None & 52 & 9 & 43 & \\
\hline Unknown & 19 & 9 & 10 & \\
\hline BMI $\left(\mathrm{kg} / \mathrm{m}^{2}\right)$ & 156 & $23.22 \pm 4.78$ & $23.89 \pm 3.23$ & 0.11 \\
\hline \multicolumn{5}{|c|}{ Preoperative measures } \\
\hline NLR & 156 & $3.18 \pm 2.88$ & $2.52 \pm 2.75$ & 0.17 \\
\hline LMR & 156 & $3.43 \pm 1.61$ & $4.20 \pm 1.69$ & $<0.01^{*}$ \\
\hline PLR & 156 & $165.91 \pm 94.37$ & $148.24 \pm 87.25$ & 0.25 \\
\hline
\end{tabular}

\section{Survival differences of ACR-low or ACR-high groups in PFS and OS}

There were significant differences in the ACR-low or ACR-high subgroups in the 3-year PFS $(63.27 \%$ vs. $20.56 \%, P<0.01)$ and OS $(48.98 \%$ vs. $14.02 \%, P<0.01)$ rates. By $\mathrm{K}-\mathrm{M}$ analyses, it was found that patients with ACR-low had a significantly worse PFS (ACR-low vs. high: $29.35 \pm 24.73 \mathrm{~m} v s .49 .81 \pm 21.43 \mathrm{~m}$, Log Rank $=35.75, \mathrm{P}<0.01$ ) and OS (ACR-low vs. high: $35.22 \pm 21.94 \mathrm{~m}$ vs. $53.28 \pm 18.98 \mathrm{~m}$, Log Rank = 29.68, $P<0.01$ ) than those with ACR-high (Fig. 3).

\section{Univariate and multivariate analyses of the prognostic factors for PFS and OS}

Univariate tests indicated that invasive depth, tumor diameter, node involvement, positive node number, distant metastasis, TNM stage, NLR, LMR and ACR were significant prognostic factors for PFS and OS (additional plus age and histological grade) (Table 2), and when all these factors were included in multivariate tests, the results indicated that the ACR was an independent prognostic factor for both PFS $(\mathrm{HR}=0.31,95 \% \mathrm{Cl}: 0.17-0.56, \mathrm{P}<0.01)$ and $\mathrm{OS}(\mathrm{HR}=0.33,95 \% \mathrm{Cl}: 0.16-0.66, \mathrm{P}<0.01)$ (Table 3$)$. 
Page $7 / 18$ 
Table 2

Univariate and multivariate analyses of different parameters for PFS

Univariate

P $\quad$ HR $\quad 95 \% \mathrm{Cl}$

Age (years)

$<60$

$\geq 60$

Gender

Female

Male

Tumor location

Right

Left

0.26

1.51

$0.74-3.10$

Histological grade

Well + moderate $\quad 1$

$\begin{array}{llll}\text { Poor } & 0.15 & 1.61 & 0.84-3.06\end{array}$

Invasive depth

\begin{tabular}{|c|c|c|c|c|c|c|}
\hline $\mathrm{T}_{1+2}$ & 1 & & & 1 & & \\
\hline $\mathrm{T}_{3+4}$ & $\begin{array}{l}< \\
0.01^{*}\end{array}$ & 5.81 & $1.81-18.67$ & $0.04^{*}$ & 3.34 & $1.02-11.01$ \\
\hline
\end{tabular}

\section{Tumor diameter}

(cm)

$\begin{array}{llll}<4 & 1 & & \\ \geq 4 & 0.13 & 1.56 & 0.88-2.79\end{array}$

Node involvement

$\begin{array}{ll}\mathrm{N}_{0} & 1\end{array}$

$\begin{array}{llll}\mathrm{N}_{1+2} & 0.03^{*} & 1.86 & 1.08-3.21\end{array}$

$\begin{array}{llllll}\text { Positive nodes no. } \quad<\quad & 1.11 & 1.06-1.16 & 0.03^{*} & 1.05 & 1.01-1.10\end{array}$
Multivariate

$\begin{array}{lll}P & H R & 95 \% \mathrm{Cl}\end{array}$


Univariate

\section{Distant metastasis}

Yes 1

No

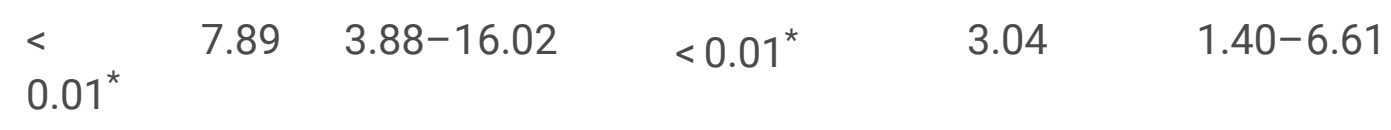

\section{TNM stage}

$\begin{array}{llll}I+I & 1 & & \\ I I+I V & < & 2.19 & 1.26-3.80\end{array}$

\section{Adjuvant therapies}

Received 1

$\begin{array}{llll}\text { None }+ \text { Unknow } \quad 0.07 & 1.69 & 0.96-3.00\end{array}$

$\begin{array}{llll}\text { BMI }\left(\mathrm{kg} / \mathrm{m}^{2}\right) & 0.39 & 0.97 & 0.89-1.05\end{array}$

\section{Preoperative measures}

$\begin{array}{lllllll}\text { NLR } & < & 1.09 & 1.03-1.15 & <0.01^{*} & 1.09 & 1.03-1.16 \\ \text { LMR } & 0.01^{*} & & & & & \\ & < & 0.77 & 0.64-0.93 & & & \\ \text { PLR } & 0.01^{*} & & & & & \\ \text { ACR } & 0.17 & 1.00 & 1.00-1.00 & & & \\ <5.98 & & & & & & \\ \geq 5.98 & 1 & & & & & \\ & < & 0.21 & 0.12-0.37 & <0.01^{*} & 0.31 & 0.17-0.56\end{array}$

* with significant statistical difference

Table 3. Univariate and multivariate analyses of different parameters for PFS 


\section{Univariate}

P $\quad$ HR $\quad 95 \% \mathrm{Cl}$

\section{Multivariate}

P
HR

$95 \% \mathrm{Cl}$

Age (years)

$\begin{array}{llll}<60 & 1 & & \\ \geq 60 & 0.02^{*} & 2.28 & 1.13-4.59\end{array}$

Gender

Female

1

Male

0.45

1.30

$0.66-2.57$

Tumor location

Right

$\begin{array}{llll}\text { Left } & 0.37 & 1.45 & 0.64-3.29\end{array}$

Histological grade

Well+moderate

1

Poor

$0.04^{\star} \quad 2.02 \quad 1.01-4.06$

Invasive depth

$\begin{array}{ll}\mathrm{T}_{1+2} & 1\end{array}$

$\begin{array}{llll}\mathrm{T}_{3+4} & 0.01^{*} & 13.49 & 1.85-98.31\end{array}$

$\begin{array}{llll}\text { Tumor diameter } \quad 0.02^{*} & 1.16 & 1.03-1.30\end{array}$

(cm)

$0.02^{*} \quad 1.16 \quad 1.03-1.30$

$\begin{array}{llll}\otimes 4 & 1 & & \\ \geq 4 & 0.03 & 2.19 & 1.07-4.50\end{array}$

Node involvement

$\mathrm{N}_{0}$ 1

$\mathrm{N}_{1+2}$

$0.01^{*} \quad 2.21 \quad 1.17-4.17$

Positive nodes no.

$<0.01^{*} \quad 1.14 \quad 1.09-1.20$

$<0.01^{*}$

1.08

$1.02-1.13$

Distant metastasis

Yes

No
1

$<0.01^{*}$

8.71

$4.01-18.94$

1

$<0.01^{*}$

4.41

$1.92-10.10$ 


\section{TNM stage}

$1+1 \mid$ 1

II+IV

$<0.01^{*} \quad 2.57 \quad 1.34-4.89$

\section{Adjuvant therapies}

\begin{tabular}{llll} 
Received & 1 & & \\
\hline None+Unknow & 0.43 & 1.30 & $0.68-2.48$ \\
\hline BMl $\left(\mathrm{kg} / \mathrm{m}^{2}\right)$ & 0.40 & 0.96 & $0.88-1.05$
\end{tabular}

\section{Preoperative measures}

$\begin{array}{lllllll}\text { NLR } & <0.01^{*} & 1.10 & 1.04-1.17 & <0.01^{*} & 1.10 & 1.03-1.18 \\ \text { LMR } & 0.03^{*} & 0.79 & 0.63-0.98 & & & \\ \text { PLR } & 0.23 & 1.00 & 1.00-1.01 & & & \end{array}$

\section{ACR}

$\varangle 5.98$ 1

$\geq 5.98$

$<0.01^{*}$

0.20

$0.10-0.38$

$<0.01^{*}$

0.33

$0.16-0.66$

*with significant statistical difference

\section{Discussion}

In the present study, we found that ACR was a useful prognostic marker in CRC, and patients with a relatively low preoperative ACR had a worse prognosis than those with a high preoperative ACR. The prognostic efficacy of ACR was significantly better than that of individual ALB, CEA and other systemic inflammation markers and was an independent risk factor for survival. To the best of our knowledge, this is the first report concerning the role of ACR in cancer.

Previously, both individual ALB and CEA were reported as conventional prognostic markers in CRC. In a study which included 69 nonmetastatic and 57 metastatic CRC patients, Wei et al. found that ALB was significantly reduced in metastatic patients and was an independent prognostic factor for PFS [10]. Li et al. studied 312 stage I-III CRC patients and found that low ALB correlated with shortened PFS and OS [11]. In line with these results, Almasaudi et al. performed a study on 795 CRC patients and found that hypoalbuminemia was associated with poorer cancer-specific survival and OS [35]. Lakemeyer et al. conducted a study with 1487 staged I-IV CRC and found that an elevated CEA $(>5 \mathrm{ng} / \mathrm{mL})$ could predict significantly worse OS [36]. Additionally, Egenvall et al. carried out a study on 2509 staged II-III CRC and found that an elevation of CEA ( $>5 \mathrm{ng} / \mathrm{mL}$ ) either before or after treatment could predict an increased risk of recurrence as well as cancer-specific mortality and OS[37]. In addition, Beom et al. studied 2021 staged 
I-III CRC with a normal preoperative CEA level $(<5 \mathrm{ng} / \mathrm{mL})$ and demonstrated that a relatively high CEA (> $2.1 \mathrm{ng} / \mathrm{mL}$ ) correlated with significantly poor disease-free survival (DFS) [27]. Interestingly, all these studies consistently indicated that a lower ALB or a relatively higher CEA could be associated with poor survival in patients, which could be partially interpreted as equal to a lower ACR in our study. Additionally, as indicated in previous studies, a lower ALB was more commonly found in cases with metastasis[10, 38] or large tumor diameter and advanced T stages [38-40]. The significant differences in invasive depth, tumor diameter and distant metastasis in the ACR-low and ACR-high subgroups in our study were also in line with these results to some extent.

Furthermore, serum albumin levels have long been regarded as a reflection of nutritional status in patients, and serum albumin metabolism could be significantly altered in the context of cancer. Except for those who underwent surgery that presented with a decreased synthesis rate of ALB [41], the generation of ALB could be purely suppressed by the activation of a series of inflammatory cytokines, including IL-1, IL-6 and TNFa, in cancer patients [18,35, 42]. Notably, CEA was found to be positively associated with IL$6[43,44]$. Based on these results, it was plausible that patients with a high preoperative CEA could have a low ALB (equal to a low ACR in our study) in CRC. Additionally, since a high CEA was produced by drugresistant cancer cells in $\mathrm{CRC}[45]$, these patients were also likely to have a poor prognosis.

Notably, individual ALB or CEA was insufficient for their prognostic efficacy in CRC, particularly when compared with other markers. For example, in Li et al.'s study, the AUCs for PFS and OS of ALB were 0.644 and 0.611 , respectively, and ALB alone was not an independent prognostic factor when compared to PLR[11]; similarly, Artaç $M$ et al. studied 90 metastatic CRC patients treated with the bevacizumab plus FOLFIRI regimen and found that ALB was not a risk factor for PFS when compared with NLR[46]. For CEA, Björkman $\mathrm{K}$ et al. investigated 322 staged I-IV CRC and found that CA125 was superior to CEA in predicting disease-specific survival (DSS)[47]. In recent years, some authors have tried to combine ALB or CEA with other markers to further improve their prognostic efficacy. For ALB, Matsuoka et al. collected 133 stage III CRC and explored the prognostic value of pre- and postoperative CAR, which was found to be an independent prognostic factor for recurrence-free survival and OS, but its AUC was only 0.63 and was the highest of the assessed markers (NLR, PLR) [48]. Yamamoto et al. studied 523 staged I-IV CRC and combined ALB with cholinesterase as a new prognostic indicator. The results suggested that patients in both ALB and cholinesterase high groups would have a better DSS than others; however, the AUCs of the combined groups were not compared with individual ALB (0.71) and cholinesterase (0.69) in their study[22]. Some authors tried to combine CEA with Ki-67 [49], p53 [50], tumor budding [51], CD44v6 [52], peritoneal carcinomatosis index [53], D-dimer [54] and NLR[55] to generate new prognostic indicators, but these studies did not compare the AUC of the new indicators with individual CEA. In our study, we found that ACR displayed the largest AUC when compared with individual ALB, CEA and NLR, LMR, PLR both for PFS and OS, which supports its priority in prognosis prediction in CRC.

The present study has some limitations. First, it was retrospectively conducted at a single center, and the relatively small sample size may attenuate the statistical power and lead to biased findings. Second, other factors could also influence PFS and OS. For example, some of the patients, including those with 
stage II disease with high recurrence or metastasis risks or those with stage III-IV disease, will accept subsequent therapies after surgery. Nonetheless, additional studies with increased cases could resolve these drawbacks and confirm the results of our study in the future.

\section{Conclusion}

Overall, our results indicated for the first time that ACR was a robust prognostic factor in CRC, and patients with a relatively low preoperative ACR had significantly worse survival.

\section{Declarations}

\section{Conflict of interests}

The authors have no conflicts of interest to declare.

\section{Acknowledgements}

The study was conducted with grants from the Chinese Natural Science Foundation (grant no. 81873215 and 81503391).

\section{References}

1. Sung H, Ferlay J, Siegel RL, et al. Global cancer statistics 2020: GLOBOCAN estimates of incidence and mortality worldwide for 36 cancers in 185 countries. CA Cancer J Clin. 2021;71:209-49.

2. Chen W, Zheng R, Baade PD, et al. Cancer statistics in China, 2015. CA Cancer J Clin. 2016;66:11532.

3. Markowitz SD, Bertagnolli MM. Molecular origins of cancer: Molecular basis of colorectal cancer. N Engl J Med. 2009;361:2449-60.

4. Rothschild MA, Oratz M, Schreiber SS. Regulation of albumin metabolism. Annu Rev Med. 1975;26:91-104.

5. Nazha B, Moussaly E, Zaarour M, et al. Hypoalbuminemia in colorectal cancer prognosis: Nutritional marker or inflammatory surrogate? World J Gastrointest Surg. 2015;7:370-7.

6. Onate-Ocana LF, Aiello-Crocifoglio V, Gallardo-Rincon D, et al. Serum albumin as a significant prognostic factor for patients with gastric carcinoma. Ann Surg Oncol. 2007;14:381-9.

7. Jin Y, Zhao L, Peng F. Prognostic impact of serum albumin levels on the recurrence of stage I nonsmall cell lung cancer. Clinics. 2013;68:686-93.

8. Ataseven B, du Bois A, Reinthaller A, et al. Pre-operative serum albumin is associated with postoperative complication rate and overall survival in patients with epithelial ovarian cancer undergoing cytoreductive surgery. Gynecol Oncol. 2015;138:560-5. 
9. Danan D, Shonka DC Jr, Selman Y, et al. Prognostic value of albumin in patients with head and neck cancer. Laryngoscope. 2016;126:1567-71.

10. Wei Y, Xu H, Dai J, et al (2018) Prognostic significance of serum lactic acid, lactate dehydrogenase, and albumin levels in patients with metastatic colorectal cancer. BioMed Res Int 2018:1804086.

11. Li Z, Xu Z, Huang Y, et al. Prognostic values of preoperative platelet-to-lymphocyte ratio, albumin and hemoglobin in patients with non-metastatic colon cancer. Cancer Manag Res. 2019;11:3265-74.

12. Egenvall M, Morner M, Martling A, et al. Prediction of outcome after curative surgery for colorectal cancer: preoperative haemoglobin, C-reactive protein and albumin. Colorectal Dis. 2018;20:26-34.

13. Chiang JM, Chang CJ, Jiang SF, et al (2017) Pre-operative serum albumin level substantially predicts post-operative morbidity and mortality among patients with colorectal cancer who undergo elective colectomy. Eur J Cancer Care 26(2).

14. Toiyama $\mathrm{Y}$, Yasuda $\mathrm{H}$, Ohi $\mathrm{M}$, et al. Clinical impact of preoperative albumin to globulin ratio in gastric cancer patients with curative intent. Am J Surg. 2017;213:120-6.

15. Shibutani M, Maeda K, Nagahara $\mathrm{H}$, et al. The pretreatment albumin to globulin ratio predicts chemotherapeutic outcomes in patients with unresectable metastatic colorectal cancer. BMC Cancer. 2015;15:347.

16. Zhang L, Qin S, Chen H, et al. Diagnostic values of the prealbumin-to-fibrinogen, albumin-tofibrinogen, and monocyte-to-lymphocyte ratios in gastric cancer. Ann Clin Lab Sci. 2021;51:385-92.

17. Ishizuka $M$, Nagata $H$, Takagi $K$, et al. Clinical significance of the c-reactive protein to albumin ratio for survival after surgery for colorectal cancer. Ann Surg Oncol. 2016;23:900-7.

18. Shibutani M, Maeda K, Nagahara H, et al. Prognostic significance of the preoperative ratio of creactive protein to albumin in patients with colorectal cancer. Anticancer Res. 2016;36:995-1001.

19. Shibutani M, Nagahara H, Fukuoka T, et al. Prognostic significance of the c-reactive protein-toalbumin ratio in patients with metastatic colorectal cancer treated with trifluridine/thymidine phosphorylase inhibitor as later-line chemotherapy. Anticancer Res. 2019;39:1051-7.

20. Guo M, Sun T, Zhao Z, et al. Preoperative platelet to albumin ratio predicts outcome of patients with non-small-cell lung cancer. Ann Thorac Cardiovasc Surg. 2021;27:84-90.

21. Tawfik B, Mokdad AA, Patel PM, et al. The neutrophil to albumin ratio as a predictor of pathological complete response in rectal cancer patients following neoadjuvant chemoradiation. Anticancer Drugs. 2016;27:879-83.

22. Yamamoto $\mathrm{M}$, Saito $\mathrm{H}$, Uejima $\mathrm{C}$, et al. Combination of serum albumin and cholinesterase levels as prognostic indicator in patients ith colorectal cancer. Anticancer Res. 2019;39:1085-90.

23. Gold P, Freedman SO. Demonstration of tumor-specific antigens in huamn colonic carcinoma by immunological tolerance and absorption techniques. J Exp Med. 1965;121:439-62.

24. Goldstein MJ, Mitchell EP. Carcinoembryonic antigen in the staging and follow-up of patients with colorectal cancer. Cancer Invest. 2005;23:338-51. 
25. Becerra AZ, Probst CP, Tejani MA, et al. Evaluating the prognostic role of elevated preoperative carcinoembryonic antigen levels in colon cancer patients: results from the national cancer database. Ann Surg Oncol. 2016;23:1554-61.

26. Huh JW, Kim CH, Lim SW, et al. Factors predicting long-term survival in colorectal cancer patients with a normal preoperative serum level of carcinoembryonic antigen. J Cancer Res Clin Oncol. 2013;139:1449-55.

27. Beom SH, Shin SJ, Kim CG, et al. Clinical significance of preoperative serum carcinoembryonic antigen within the normal range in colorectal cancer patients undergoing curative resection. Ann Surg Oncol. 2020;27:2774-83.

28. Cui M, Xu R, Yan B. A persistent high neutrophil-to-lymphocyte ratio predicts poor prognosis in patients with colorectal cancer undergoing resection. Mol Clin Oncol. 2020;13(5):63.

29. Xu RYJ, Li F, et al (2020) Postoperative fasting blood glucose predicts prognosis in stage I-III colorectal cancer patients undergoing resection. Gastroenterol Res Pract 2020:2482409.

30. Li X, An B, Ma J, et al. Prognostic value of the tumor size in resectable colorectal cancer with different primary locations: a retrospective study with the propensity score matching. $\mathrm{J}$ Cancer. 2019;10:313-22.

31. Li H, Zhao Y, Zheng F. Prognostic significance of elevated preoperative neutrophil-to-lymphocyte ratio for patients with colorectal cancer undergoing curative surgery: A meta-analysis. Medicine. 2019;98:e14126.

32. Tan D, Fu Y, Tong W, et al. Prognostic significance of lymphocyte to monocyte ratio in colorectal cancer: A meta-analysis. Int J Surg. 2018;55:128-38.

33. Min GT, Wang $\mathrm{YH}$, Yao N, et al. The prognostic role of pretreatment platelet-to-lymphocyte ratio as predictors in patients with colorectal cancer: a meta-analysis. Biomark Med. 2017;11:87-97.

34. Eisenhauer EA, Therasse P, Bogaerts $\mathrm{J}$, et al. New response evaluation criteria in solid tumours: revised RECIST guideline (version 1.1). Eur J Cancer. 2009;45:228-47.

35. Almasaudi AS, Dolan RD, Edwards CA, et al (2020) Hypoalbuminemia reflects nutritional risk, body composition and systemic inflammation and is independently associated with survival in patients with colorectal cancer. Cancers 12:1986.

36. Lakemeyer L, Sander S, Wittau M, et al. Diagnostic and prognostic value of CEA and CA19-9 in colorectal cancer. Diseases. 2021;9:21.

37. Egenvall $M$, Martling $A$, Veres $K$, et al. No benefit of more intense follow-up after surgery for colorectal cancer in the risk group with elevated CEA levels-An analysis within the COLOFOL randomized clinical trial. Eur J Surg Oncol. 2021;47:2053-9.

38. Thirunavukarasu P, Talati C, Munjal S, et al. Effect of Incorporation of pretreatment serum carcinoembryonic antigen levels into AJCC staging for colon cancer on 5-year survival. JAMA Surg. 2015;150:747-55.

39. Ouyang $X$, Dang $Y$, Zhang $F$, et al. Low serum albumin correlates with poor survival in gastric cancer patients. Clin Lab. 2018;64:239-45. 
40. Ozawa H, Kotake K, Hosaka M, et al. Incorporation of serum carcinoembryonic antigen levels into the prognostic grouping system of colon cancer. Int J Colorectal Dis. 2017;32:821-9.

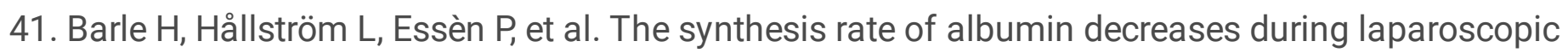
surgery. Clin Physiol Funct Imaging. 2004;24:91-5.

42. Don BR, Kaysen G. Serum albumin: relationship to inflammation and nutrition. Semin Dial. 2004; 17:432-7.

43. Belluco C, Nitti D, Frantz M, et al. Interleukin-6 blood level is associated with circulating carcinoembryonic antigen and prognosis in patients with colorectal cancer. Ann Surg Oncol. 2000;7:133-8.

44. Nakagoe T, Tsuji T, Sawai T, et al. The relationship between circulating interleukin- 6 and carcinoembryonic antigen in patients with colorectal cancer. Anticancer Res. 2003;23:3561-4.

45. Lee HC, Ling QD, Yu WC, et al. Drug-resistant colon cancer cells produce high carcinoembryonic antigen and might not be cancer-initiating cells. Drug Des Devel Ther. 2013;7:491-502.

46. Artac M, Uysal M, Karaagac M, et al. Prognostic impact of neutrophil/lymphocyte ratio, platelet count, crp, and albumin levels in metastatic colorectal cancer patients treated with FOLFIRIbevacizumab. J Gastrointest Cancer. 2017;48:176-80.

47. Björkman K, Mustonen H, Kaprio T, et al. CA125: A superior prognostic biomarker for colorectal cancer compared to CEA, CA19-9 or CA242. Tumour Biol. 2021;43:57-70.

48. Matsuoka H, Ando K, Hu Q, et al. Postoperative C-reactive protein/albumin ratio is a biomarker of risk of recurrence and need for adjuvant chemotherapy for stage III colorectal cancer. Int J Clin Oncol. 2020;25:1318-26.

49. Peng Y, Wang L, Gu J. Elevated preoperative carcinoembryonic antigen (CEA) and Ki67 is predictor of decreased survival in IIA stage colon cancer. World J Surg. 2013;37:208-13.

50. Nasif WA, Lotfy M, El-Sayed IH, et al. Implications of CEA and p53 overexpression in the poor prognosis of colorectal cancer. Med Oncol. 2006;23:237-44.

51. Du C, Xue W, Dou F, et al. Use of a combination of CEA and tumor budding to identify high-risk patients with stage II colon cancer. Int J Biol Markers. 2017;32:e267-73.

52. Chen L, Jiang B, Wang Z, et al. Combined preoperative CEA and CD44v6 improves prognostic value in patients with stage I and stage II colorectal cancer. Clin Transl Oncol. 2014;16:285-92.

53. Kozman MA, Fisher OM, Rebolledo BJ, et al. CEA to peritoneal carcinomatosis index (PCl) ratio is prognostic in patients with colorectal cancer peritoneal carcinomatosis undergoing cytoreduction surgery and intraperitoneal chemotherapy: A retrospective cohort study. J Surg Oncol. 2018;117:725-36.

54. Ojima Y, Harano M, Sumitani D, et al. Prognostic value of preoperative carcinoembryonic antigen and D-dimer concentrations in patients undergoing curative resection for colorectal cancer. Surg Today. 2021;51:1108-17. 
55. Kim H, Jung HI, Kwon SH, et al. Preoperative neutrophil-lymphocyte ratio and CEA is associated with poor prognosis in patients with synchronous colorectal cancer liver metastasis. Ann Surg Treat Res. 2019;96:191-200.

\section{Figures}

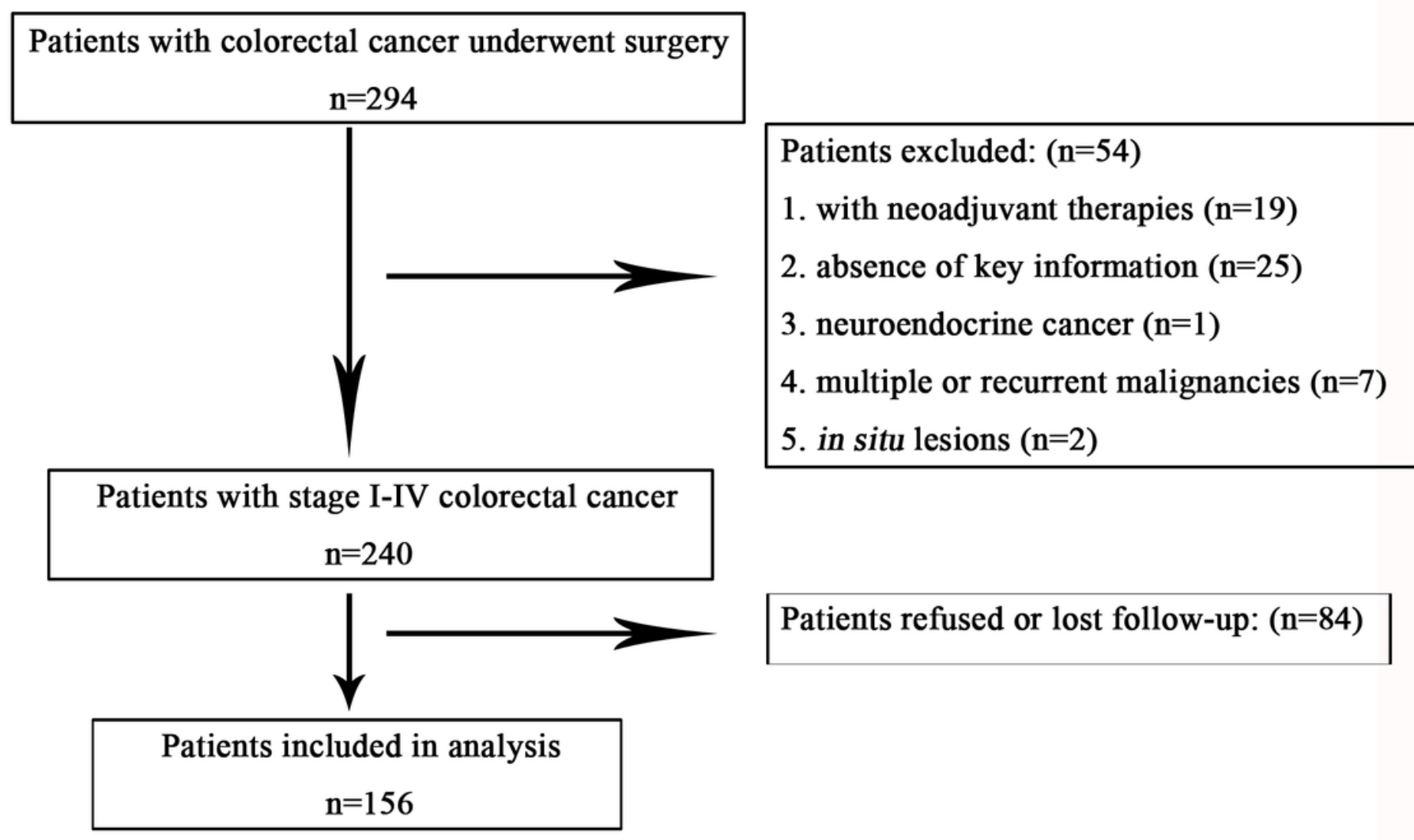

Figure 1

Flow diagram of the study. 

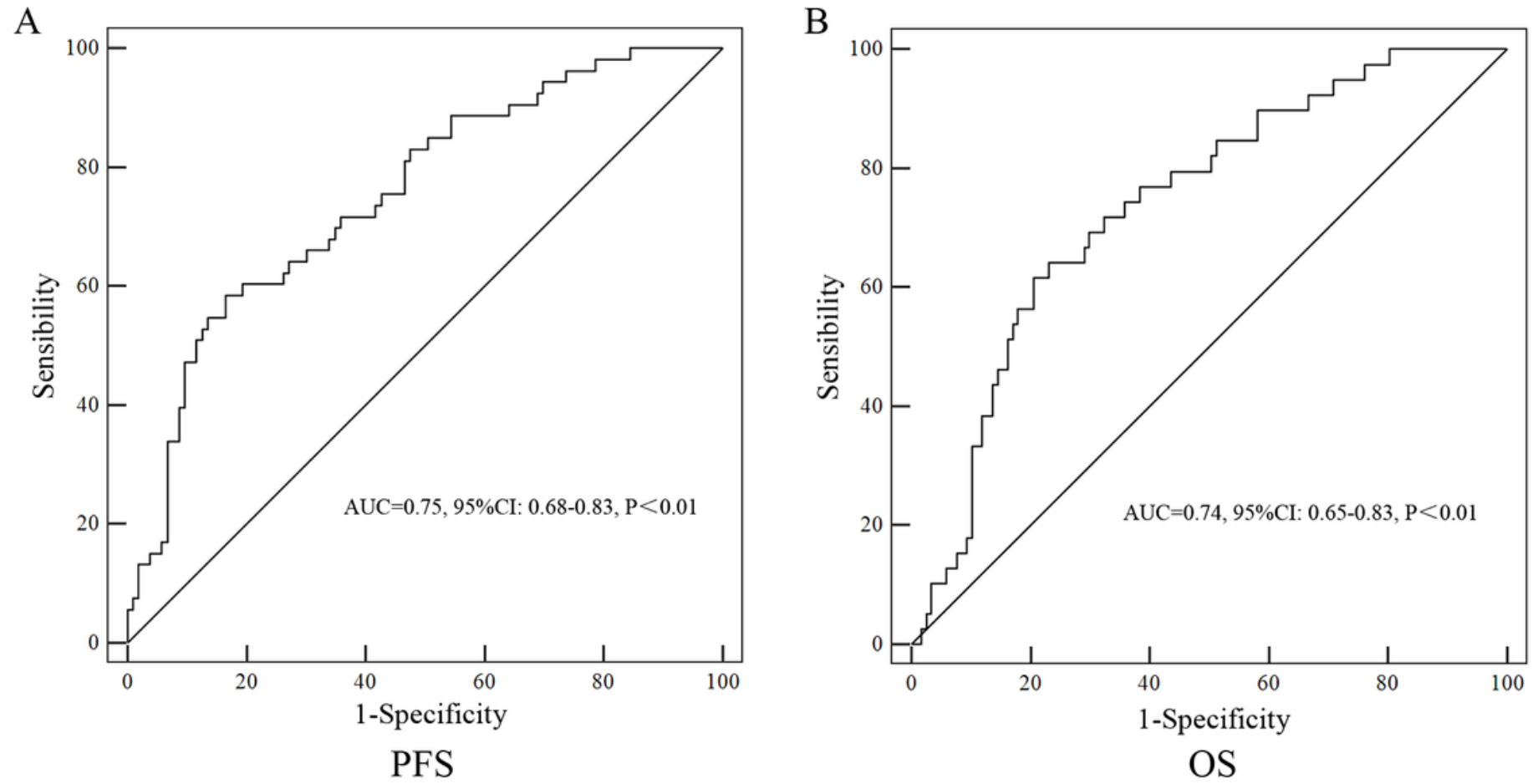

Figure 2

ROC tests of ACR for PFS and OS
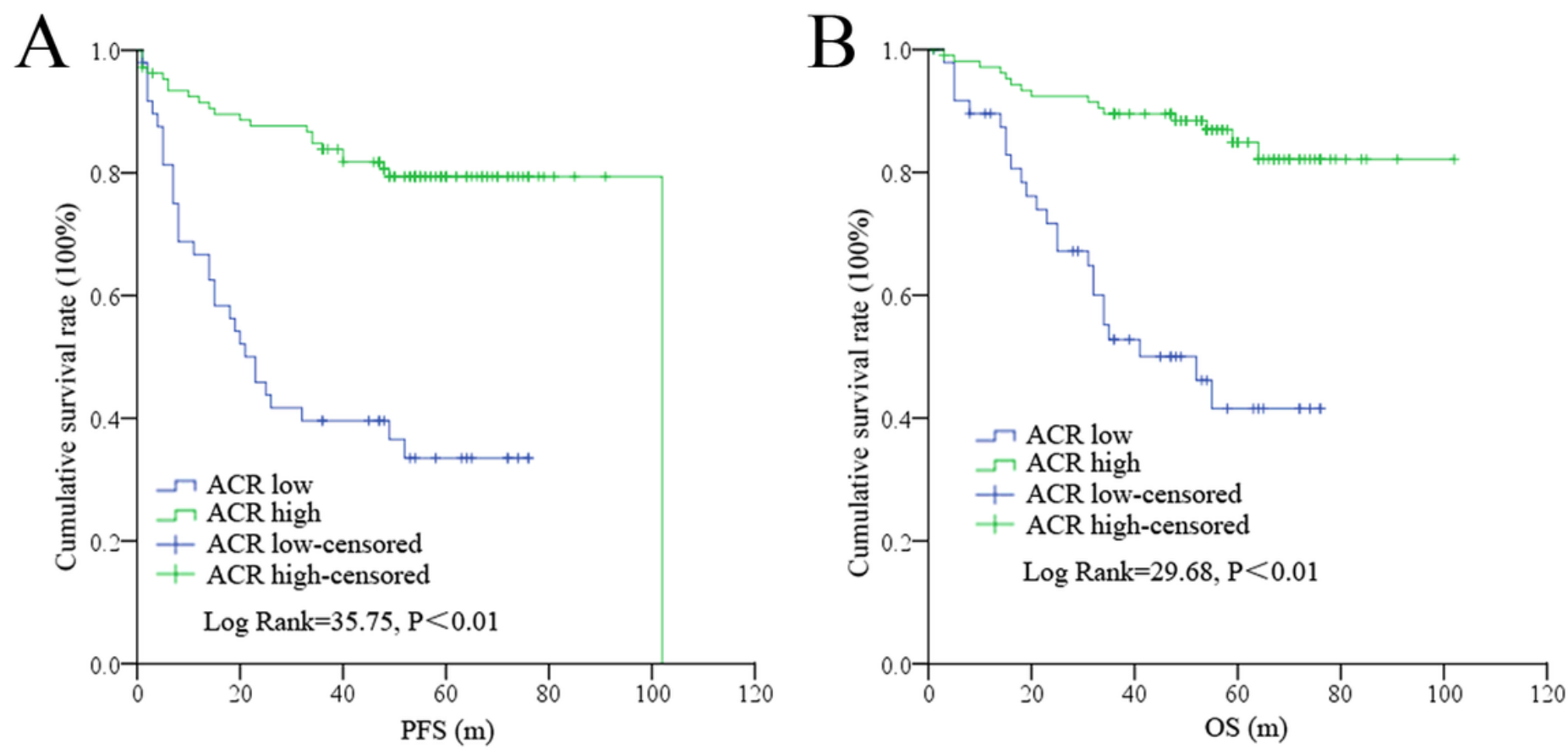

\section{Figure 3}

Impact of ACR-low or -high on PFS and OS in the patients. 